\title{
Vasodilatation in the human parotid gland after post-ganglionic sympathetic denervation
}

\author{
J. A. SIMPSON AND A. I. S. MACPHERSON \\ From the Neurological Unit, Northern General Hospital, and the Department \\ of Surgery, Royal Infirmary, Edinburgh
}

The salivary glands have a fascination for the physiologist because of their favourable position for observation of a gland which is under autonomic control and which is readily accessible to cannulation. Despite this, there is still uncertainty about thsir innervation. There is a parasympathetic and a sympathetic nerve supply but the exact role of each is debatable and indeed there may be species differences. Spontaneous secretion is prominent in the parotid gland of the sheep and the sublingual gland of the cat and is probably present in human beings though small in amount ard perhaps inter- mittent. Adrenaline from the adrenal medull certainly has an action on the salivary gland and the possibility of other hormonal effects cannot be excluded. None the less, the control of salivation is mainly neurogenic. The difficulty is to determine the role of each nerve supply and it is not even certaify that they have been fully identified.

Present views on the nerve supply of the humas parotid gland are represented by Figure 1. Thie parasympathetic fibres pass from the extracranial glossopharyngeal nerve (IX) back into the skull boj Jacobson's nerve to the tympanic plexus in the

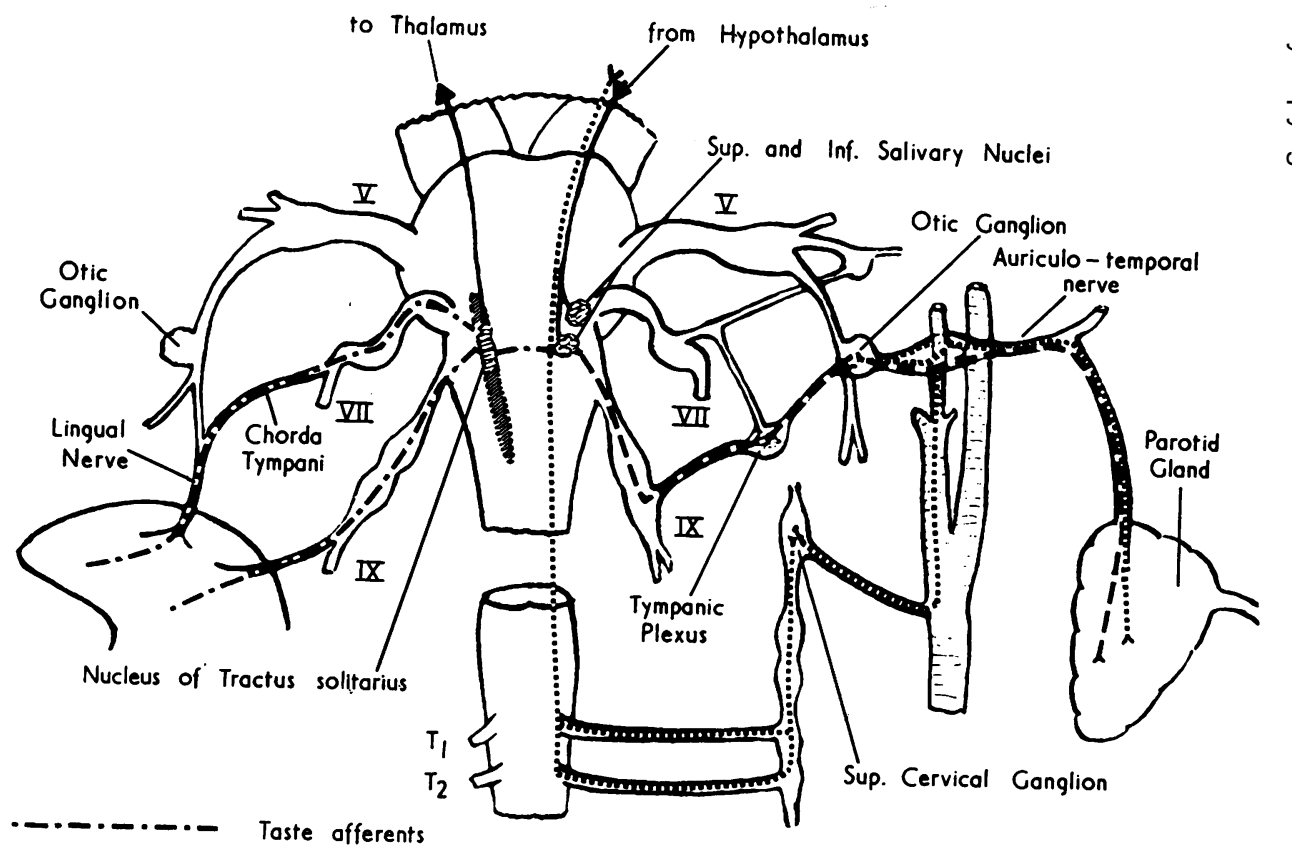

FIG. 1. Nerve supply of the parotid gland. The para sympathetic and sympathetic innervation is shown on tho diagram and the principal taste pathways on the left. The pathways for appreciation of taste and for the salivari reflex are uncertain after the nucleus of the tractus solitariust. 
middle ear, and so to the lesser superficial petrosal nerve which leaves the skull by the foramen ovale to the otic ganglion. Post-ganglionic fibres enter the auriculo-temporal nerve which supplies the parotid gland. The auriculo-temporal nerve also carries the sympathetic supply which reaches it from the superior cervical ganglion by a plexus around the external carotid artery and its branches. The preganglionic fibres emerge from the first and second thoracic segments of the spinal cord and ascend the cervical sympathetic chain to relay in the superior cervical ganglion.

Stimulation of the glossopharyngeal nerve, a cholinergic nerve, causes secretion of salivary juice from the parotid gland which is accompanied by vasodilatation. Stimulation of the sympathetic adds to the secretion in some animals though in man the parotid may not take part in this response (Folkow and Laage-Hellman, 1960). It is not yet certain whether this is a true secretagogic effect or due to vascular changes in the gland. Blood vessels in the parotid constrict for a short time then there is a long phase of dilatation. The myoepithelial basket cells contract on sympathetic stimulation, expressing saliva from the acini, and the sympathetic control may be responsible for contraction of the salivary ducts.

One of the most difficult questions is the relation between the salivary secretion and the blood flow. The present paper does not lead to a definitive conclusion but may contribute to a more recent concept, namely that vasodilatation in the gland is secondary to the salivary secretion. It has been recognized for a century that reflex stimulation of salivation is accompanied by vasodilatation in the glands but these always occur together. Many have considered that the increased vascularity is responsible for the flow of saliva. Ungar and Parrot (1936) started a new approach when they suggested that the vasodilator activity of saliva shown by earlier workers might be due to a local hormone then called kallikrein. Hilton and Lewis (1956) concluded from a series of experiments designed to elucidate the relation between cholinergic nerve supply and vasodilatation that the latter is due to an intracellular enzyme which escapes from the gland cells during activity to act upon a protein in the interstitial fluid, forming a vasodilator polypeptide which they consider to be bradykinin (also named kallidin by other workers). The intracellular enzyme is considered to be kallikrein. This theory would account for the postconstriction vasodilatation following sympathetic stimulation as well as for the parasympathetic effect. One difficulty is that the secretory response to parasympathetic stimulation can be blocked with atropine without affecting the vasodilatation. So far as the sympathetic is concerned, there is some evidence in the cat for the presence of sympathetic vasodilator fibres whose secretion is not blocked by atropine (Emmelin, 1955).

The case presented here is worth describing as a rare syndrome not well recognized, but it has also provided an opportunity to test the bradykinin hypothesis in a human being.

\section{CASE REPORT}

An unmarried woman then aged 24 attended the Neurological Unit in 1954 complaining that for three months she had suffered from headache and difficulty with vision because she could not change focus rapidly. She had noticed that the left pupil was enlarged during this period. Six months later the right pupil suddenly enlarged and vision of that eye became blurred. Examination showed myotonic pupils and myotonia of lens accommodation associated with absence of knee and ankle jerks (HolmesAdie syndrome). It was found that procaine block of the stellate ganglion relieved her symptoms. Pilocarpine drops and lenses to assist reading did not relieve symptoms and she was unable to continue with her clerical work. In view of the response to sympathetic block, bilateral stellate ganglionectomy was carried out by Mr. C. W. A. Falconer at the Western General Hospital in August 1956 with remarkable benefit. Reading improved from $\mathrm{J} 8$ to $\mathrm{J} 1$ types. There was a bilateral Horner's syndrome with loss of sweating over the face and down to the $\mathrm{C} 7$ segments of the upper limbs, but excluding the ulnar borders of the forearms.

In November 1959 visual blurring in the left eye and headache returned. Examination showed dilatation of the left pupil. The pupil dilated further when cocaine hydrochloride $(3 \%)$ was instilled into the conjunctival sac but this response was abolished when the left stellate ganglion was infiltrated with procaine. It was concluded that there was no end-organ hypersensitivity but that there must have been re-innervation of the pupil and ciliary muscle by sympathetic fibres. She was so enthusiastic about the value of her first operation that she pressed for further sympathectomy. Mr. Falconer was travelling abroad and kindly invited A.I.S.M. to take over the surgical care of the patient. She was admitted to Edinburgh Royal Infirmary and a further operation was performed on 26 April 1960. The left superior cervical sympathetic ganglion was dissected with all of its connexions up to the base of the skull where it was avulsed. The chain was also followed downwards for about $1 \frac{1}{2}$ in. and divided. Dr. A. Wynn-Williams reported that the tissue removed consisted mostly of nerve ganglion tissue which showed some fibrosis but was otherwise normal and rich in normal-looking nerve cells. Some apparently normal nerve was also present.

The immediate post-operative course was uneventful. Visual accommodation was subjectively improved and the patient no longer had difficulty with reading though distant vision was a little blurred and her eyes watered in bright sunlight. Fourteen days after operation the mucous membrane of the left side of the nose became 
congested and at the same time she began to complain of pain in the region of the left parotid gland, worse when eating. There were no signs of inflammation, no rise of temperature, and a course of penicillin did not help.

The pain started as soon as food entered her mouth, whether it was fluid or solid, and was more severe with hot food. There was no sweating of the overlying skin but flushing of the left side of the face was marked. The pain did not seem to depend on chewing though it was made worse by movements of the jaws, and investigation of the temporo-mandibular joints did not reveal any abnormality. The left parotid and submandibular regions were tender. It was considered that the pain was evoked reflexly in the left parotid gland (and possibly the submandibular gland) by the stimulus of food entering the mouth. Various investigations were made to confirm this opinion and drugs were used without lasting benefit. These measures are detailed below. Finally it was decided to depress parotid gland function by radiotherapy. Dr. J. McLelland gave a single treatment of 600 rads on 27 July 1961. There was reactionary oedema which was very painful for two days but thereafter she had considerable relief. She was able to eat freely and painlessly without requiring drugs but a little submandibular ache remained.

\section{PHYSIOLOGICAL INVESTIGATIONS}

The relationship between pain and reflex stimulation of the parotid gland was immediately confirmed when a few drops of lemon juice were placed on the patient's tongue and the spurt of saliva from all salivary ducts indicated the absence of duct obstruction. It was concluded that pain was likely to be due to distension of the gland during activity, either because of hypersecretion or vascular congestion. In view of the current belief that sympathetic fibres are capable of augmenting salivary secretion it seemed possible that hypersecretion was occurring as a manifestation of denervation hypersensitivity. The following experiments were designed to test these hypotheses.

ANAESTHETIZATION OF TASTE RECEPTORS Several local anaesthetics were tried as follows:-

Amethocaine $\mathrm{HCl}$ lozenges (Decicain) made the mouth numb but did not prevent reflex salivation and pain was unaffected. Anaesthesia was probably incomplete and it would not, of course, block 'psychic' secretion.

Lignocaine $\mathrm{HCl}(4 \%)$ applied locally did not abolish taste completely but blocked the salivary and pain response to lemon juice.

BLOCK CONFINED TO CHOLINERGIC RECEPTORS A group of drugs which would reduce salivation was tried as follows:-

Atropine sulphate, grain $1 / 100$, injected subcutaneously reduced salivation but pain on eating was only a little less.

Promethazine hydrochloride (Phenergan), 25 mg. t.i.d., by mouth had a similar effect to atropine.

Benzilonium bromide (Portyn), $10 \mathrm{mg}$. t.i.d., caused a dry mouth and slight blurring of vision. Salivation and pain were a little reduced but on testing with lemon juice there was a prompt increase in salivary secretio and immediate severe pain.

The effect of penthienate methobromide (Monodral) $5 \mathrm{mg}$. t.i.d., was similar to the other antiparasympathetic drugs, that is, some drying of the mouth with relief of pain but not sufficient to block the response to lemong juice. There was sufficient relief for three hours after eack dose to make this drug of some value.

CENTRAL AUTONOMIC BLOCK Chlorpromazine (Largactil), $25 \mathrm{mg}$. b.d., had no apparent influence on pain or sal vation. It is considered to block central sympathetic as well as parasympathetic pathways but probably at higher level than the salivary reflex. The dose used might not affect vasomotor control.

AUTONOMIC GANGLION BLOCK (SYMPATHETIC AND PARAC SYMPATHETIC) Hexamethonium bromide, $10 \mathrm{mg}$., was injected intravenously while parotid pain was present. I!̣ two and a half minutes pain was reduced and in 10 minutes it was absent. Spontaneous salivation stopped 10 minutegg after the injection but a subnormal salivary response to. lemon juice was still present. Recurrence of slight pain an $\overrightarrow{\text { A }}$ spontaneous salivation took place at 14 minutes. During this period the pupil reactions to light were abolished and response to near vision greatly reduced. It was concluded that blockade of parasympathetic ganglia was associकse with temporary relief of pain pari passu with diminis reflex salivation. The post-ganglionic sympathectofny precluded effects on the sympathetic innervation of ahe parotid gland.

BLOCK OF OTIC GANGLION Lignocaine $2 \%$, with adrôn line 1 in 80,000 (Xylocaine), was injected ( $8 \mathrm{ml}$.) into neighbourhood of the left otic ganglion with the interf $\bar{F} \overrightarrow{\mathrm{B}}$ of obtaining complete parasympathetic block of the te? parotid while any post-ganglionic sympathetic fibres still remaining would also be blocked, though it seemes unlikely that there could be any. The effect was urह equivocal. The patient could now suck acid drops of lemon juice without any pain. This was not due to spreas of lignocaine to the mandibular nerve as there was no los of sensation in the skin of the face or mucosa of the mouth. There was slight tingling of both lips on the lef? The left pupil increased in diameter but both still reacted myotonically to light.

TESTS FOR HYPERSENSITIVITY OF END-ORGANS It was considered that the unilateral dilatation of the pupil $i \overline{\mathrm{n}}$ the last experiment could be due to hypersensitivity to adrenaline in the local anaesthetic. Adrenaline hydro chloride, 1 in 1,000 , was injected intramuscularly in a dose of $0.125 \mathrm{ml}$., calculated to be the equivalent of that admin? stered with the lignocaine injection. During the period three to 10 minutes after the injection the left pupil was? little larger than the right, confirming that there was end-organ hypersensitivity. It was noted in both expers ments that no parotid pain was produced. Unfortunater. the secretion of saliva was not noted, but it may be concluded that even if the secretory cells were hype iso sensitive to adrenaline this did not provoke pain.

Edrophonium chloride (Tensilon), a short-acting drưge 
with anti-cholinesterase and parasympathomimetic properties, was injected intravenously $(10 \mathrm{mg}$.). There was a little increase in salivation but no pain. Fasciculation of eyelids occurred one minute after injection. The pupils were unaffected. This normal response makes it unlikely that cholinergic receptors were hypersensitive.

This series of experiments led to the following conclusions:-

1 Parotid pain was related only to reflex stimulation of the gland.

2 The secretion of saliva could be reduced without abolishing the pain, and conversely salivation induced by end-organ stimulation did not cause pain.

3 Hexamethonium reduced both secretion and pain, and both were stopped by blocking the salivary reflex at the otic ganglion. This incidentally also shows that pain was confined to the parotid gland and did not involve the submandibular and sublingual glands which receive their parasympathetic supply from the facial nerve via the chorda tympani.

4 There was no evidence of hypersensitivity of cholinergic or adrenergic receptors on secretory cells in the parotid gland.

\section{THE MECHANISM OF VASODILATATION}

These experiments showed that pain was related to parasympathetic activity but that it was unlikely to be due to hypersecretion of saliva. The alternative explanation that it was caused by reflex congestion of the gland seems likely since the appearance of pain was associated in time with unilateral nasal congestion and flushing of the face. It is well accepted that stimulation of the parasympathetic nerve to a salivary gland causes vasodilatation as well as secretion, and that sympathetic stimulation causes vasoconstriction which is at least sometimes followed by vasodilatation. There has been a great deal of discussion regarding the mechanism of vasodilatation since it was demonstrated by Claude Bernard (1858). This was for many years considered to prove the existence of vasodilator nerves in, at least, the chorda tympani of the dog. Carlson (1907) showed that stimulation of the sympathetic trunk in the neck of the cat usually caused marked vasodilatation in the submaxillary gland. Emmelin (1955) showed that sympathetic vasoconstriction could be dissociated from secretion but that sympathetic vasodilatation could not. The apparently contradictory reports of early investigators are well reviewed by Burgen and Emmelin (1961).

The concept of active vasodilatation by parasympathetic vasomotor nerves has never been completely accepted. Heidenhain (1872) reported that vasodilatation could still be elicited after suppression of secretion by atropine but Henderson and Loewi (1905) found that vasodilatation was reduced and suggested that it was secondary to some metabolite released by the active gland. Barcroft (1914) showed that submaxillary gland metabolism increased on stimulation of the chorda tympani even when the secretory response was blocked by atropine. In the following years the vasodilator effect of saliva was discovered and at first attributed to acetylcholine in the juice. Ungar and Parrot (1936) re-examined the problem and concluded that it was due to the presence of a local hormone, 'kallikrein'. Hilton and Lewis (1956) found that vasodilatation in the submaxillary gland of the cat was related to activity of cholinergic nerve fibres but that the agent responsible was not acetylcholine. In an important series of investigations they found that the perfusate of an active salivary gland contained a substance which caused vasodilatation (and contraction of smooth muscle) only after it had been incubated with plasma. They concluded that activity in secretor cells causes the escape of an intracellular enzyme into the interstitial fluid where it reacts with protein to form the vasodilator polypeptide bradykinin (also known as kallidin) which is removed by the lymphatics. The intracellular enzyme is identified with the kallikrein of Ungar and Parrot (1936).

The bradykinin theory would explain the vasodilatation accompanying secretion whether parasympathetic or sympathetic in origin. Nevertheless, Burgen and Emmelin (1961) feel that it is not possible to reject the concept of nerve fibres capable of producing vasodilatation without preceding salivary secretion, even if they act via kallikrein. Terroux, Sekelj, and Burgen (1959) showed that the oxygen consumption of the dog's submaxillary gland was directly related to the rate of saliva flow but the blood flow was not. Salivary flow and gland metabolic rate were depressed by atropine without affecting the vasodilator response (which in fact tended to increase with stimulation because of the lack of haemoconcentration when no fluid was withdrawn ty salivary secretory cells). It does not necessarily follow that the liberation of an intracellular enzyme when a gland is stimulated is directly correlated with the cellular oxygen consumption, but it is obvious that the bradykinin theory is not yet accepted by all authorities. We have taken the opportunity to investigate the role of bradykinin within the limits possible in a cooperative patient, assuming that pain in the parotid gland was an indication of vasodilatation within the gland.

\section{STUDIES ON BRADYKININ}

On 26 June 1961 both parotid ducts were cannulated with fine polythene tubing. Secretion was stimulated by placing lemon juice on the patient's tongue. This caused profuse salivation accompanied by severe parotid pain. Twentyfive ml. of watery saliva was obtained from the left 
(painful) gland and $10 \mathrm{ml}$. from the other. These were submitted to Dr. W. E. Brocklehurst for estimation of their content of bradykinin. Unfortunately the tube containing saliva from the painless right parotid was broken in the centrifuge. The saliva from the painful left parotid gland contained a substance which caused contraction of guinea-pig ileum equivalent to that caused by $8 \mu \mu \mathrm{g}$. $/ \mathrm{ml}$. bradykinin. The very small amount made it impracticable to characterize the substance further by testing the chymotrypsin response. The normal range for the human subject is unknown but the assay is certainly very small and does not suggest excess.

The second approach was to attempt to block the action of bradykinin on the blood vessels. It is known that salicylates and antihistamine drugs have some such effect (Collier and Shorley, 1960).

ACETYLSALICYLIC ACID Acetylsalicylic acid soluble, (B.P.), four tablets given before meals, did not prevent reflex pain.

PROMEThAZINE Promethazine, as reported above, produced no more effect than could be attributed to its anticholinergic effect. The possibility of histaminergic vasodilator nerves also seems to be excluded.

HOMOCHLORCYCLIZINE Homochlorcyclizine (SA-97 Abbott) antagonizes acetylcholine, histamine, 5-hydroxytryptamine, and the slow-reacting substance (SRS-A) of Brocklehurst (Kimura, Young, and Richards, 1960). Its activity with bradykinin is unknown but its wide spectrum of activity on tissue hormones suggested a trial. Unlabelled tablets and dummy tablets of identical appearance were obtained for trial. The active tablets contained $25 \mathrm{mg}$. of the drug. Two tablets were given thrice daily 30 minutes before meals, three days on one tablet then three days on the other. The authors did not know which tablet contained the active drug until the trial ended. The patient was asked to record her experience with meals and to try the effect of sucking an acid drop each day. She obtained no useful relief from either tablet and indeed the control tablet gave the better response.

It was concluded from these experiments that there was no evidence of excess secretion of bradykinin in saliva from the affected gland (though this requires confirmation), and that drugs believed to antagonize bradykinin did not relieve the pain experienced with reflexly stimulated salivation. On the contrary, the relief obtained was less than that from anticholinergic drugs, especially Monodral. As the latter did not relieve pain ccmpletely, despite unpleasant dryness of the mouth and visual blurring, the decision was taken to destroy the gland secretion by a small dose of radiotherapy. This reduced the severity of pain for about nine months but at the time of writing there has been some recurrence. This may suggest that vasodilatation is independent of secretory activity, and our other findings suggest that they are related only by common reflex stimulation. We find no evidence of increased bradykinin formation and there is at present no reason to $\overline{\mathbb{Z}}$ believe that post-ganglionic sympathectomy renders $\stackrel{\mathbb{D}}{=}$ the parotid gland vessels hypersensitive to the polypeptide. Our findings would be quite compatible $\frac{\varsigma}{\widehat{C}}$ with the presence of vasodilator nerves. As Barcroft (1914) has written before about this problem: 'It's is not possible on the evidence at hand either to $\frac{\hat{}}{\vec{a}}$ prove or to disprove it'.

\section{DISCUSSION}

The myotonic contraction, well known in the pupil in? patients with the Holmes-Adie syndrome, also $\frac{\bar{\sigma}}{\bar{n}}$ involves the ciliary muscle causing slowness in? accommodation. The inability to change focus $\subseteq$ rapidly when looking up from a book is a great inconvenience to a clerical worker. The time for $\vec{\circ}$ relaxation of accommodation on looking from a near: to a distant object can be accelerated by blocking the $\vec{\omega}$ cervical sympathetic nerves with local anaesthetic. The rate of relaxation is not altered, but the range of change required is smaller (Russell, 1956). For this reason bilateral stellate ganglionectomy was. carried out in 1956 and the functional result fulty justified the operations. This is a pre-gangliongc + sympathectomy and it was presumed that regenes? ation had occurred on the left side when the 1e्gitpupil dilated and accommodation was again difficudit. T Denervation hypersensitivity was excluded showing a normal response to cocaine, and a miofico response to procaine block of the cervical sy pathetic chain. At the patient's request the refte sympathetic was again cut, but this time the superiores cervical ganglion was removed and its nature confirmed by histology. (In passing, we believe this to be the only observation on that structure in the HolmesAdie syndrome.)

Fourteen days after the post-ganglionic operation, which successfully cured her visual complaint, the patient noticed congestion of the left side of theo nose and pain in the left side of the face on taking food or fluids into the mouth. She was observed to have flushing of the left side of her face. These clinical points may well be considered to argue against any local peculiarity of vasomotor supply in the salivary glands. One of us (J.A.S.) considered? that the pain was localized in the parotid gland and likened it to that occurring after sympathetico blockade by bretylium (Lowther and Turner, 1960) though the latter differs in being blocked by atropine은 (Emmelin and Engström, 1960). The investigations $>$ described in this paper were planned to explore the nature of the pain. It has been demonstrated that itn is related to reflex excitation of the parotid gland but is not directly related to its secretory activity. It is submitted that the probable explanation is a 
functional vasodilatation within the gland. Limited studies to test the hypothesis that salivary gland vasodilatation is secondary to secretory activity which releases an intracellular enzyme for the production of bradykinin have been made with inconclusive but probably negative results.

We were interested to find a report by Gardner and Abdullah (1955) of parotid pain on eating in 14 patients who had superior cervical ganglionectomy. Patients who also had a preganglionic sympathectomy on the other side had no pain on that side. Pain usually started in the second week after operation. In most of their patients it lasted for only a few seconds at the beginning of a meal, and tended to become milder in a few months though it did not disappear. They also found that atropine did not abolish the pain while depressing secretory response but that both were depressed by tetraethylammonium chloride, which acts in the same way as the hexamethonium used by us. They conclude that absence of the tonic vasoconstrictor effect of the sympathetic nerve allows excess 'parasympathetic' vasodilatation but that there is sufficient autonomous activity in the de-afferented ganglion (pre-ganglionic section) to keep parasympathetic vasodilatation within physiological bounds. Their approach to therapy was more heroic than ours. On the assumption that the autonomic balance would thereby be restored, they sectioned the glossopharyngeal nerve (IX) intracranially. In one of their cases this operation did not have the desired effect. They make the interesting suggestion that some parasympathetic fibres reach the parotid gland from the facial nerve. Reichert and Poth (1933) reached the same conclusion when they observed that section of either the glossopharyngeal nerve (intracranially) or the chorda tympani (in the middle ear) in three patients decreased the secretion from all three salivary glands. We have nothing to contribute on this aspect since the response to otic ganglion/auriculotemporal block in our case was so complete.

This unusual syndrome has points in common with the auriculo-temporal syndrome of Frey (1923), facial hyperaemia and sweating after destruction of the auriculo-temporal nerve, and the similar unilateral submental hyperaemia and sweating after damage to the chorda tympani (Uprus, Gaylor, and Carmichael, 1934). Unilateral sweating of the face followed superior cervical ganglionectomy in a patient in whom an extracranial portion of the ipsilateral glossopharyngeal nerve had been resected and the sensory root of the trigeminal nerve sectioned. The sweating response was evoked with a salivary reflex but there was no obvious flushing of the face (Wilson, 1936). Uprus et al. (1934) found that atropine abolished submental sweating but flushing 8 still occurred on eating an apple. They conclude that atropine blocks the secretory activity of the chorda tympani without impairing its vasodilator activity. Blocking the chorda tympani temporarily blocked the whole of the response on eating but cervical sympathetic block did not. Writers on these syndromes do not stress the vascular response and assume the presence of vasodilator nerves. It would be interesting to know if the vascular response occurs in the absence of the denervated salivary gland. A recent review of the auriculo-temporal syndrome by Glaister, Hearnshaw, Heffron, Peck, and Patey (1958) is introduced by the comment that 'gustatory sweating . . . may be associated with flushing of the skin in the same region' but it was minimal or absent in their cases which were all the result of excision of the parotid gland. They dismiss Frey's theory that gustatory sweating is due to a diffusion of a choline-like substance from the parotid gland 'since the syndrome (sic) may occur after total parotidectomy'. By the same token if their observation that flushing is absent from the syndrome in these cases is confirmed, it might be suggested that flushing is not caused by vasodilator nerves but by diffusion from the gland of a substance which may be bradykinin. Kidd (1955) specifically mentions ipsilateral flushing of the face after total parotidectomy and this occurred in the skin supplied by the auriculo-temporal nerve. If the nerve was sectioned the Frey syndrome was rare and was possibly associated with incomplete neurectomy. The possibility of diffusion from a salivary gland can be excluded in the case of the pain in the lower limbs which may follow lumbar sympathectomy. Tracy and Cockett (1957) noted a characteristic latency of 10 to 17 days between operation and onset of pain, and pointed out that there was excessive sweating in the painful dermatomes. Unfortunately they did not consider the possibility of end-organ hypersensitivity and do not discuss skin temperature or pilomotor changes. Nevertheless, it appears that post-sympathectomy vasodilatation and/or pain are not confined to salivary glands. Though we are not prepared to reject the possibility of a hormonal vasodilator, our study supports the older concept of a parasympathetic vasodilator nerve supply to the parotid gland.

\section{SUMMARY}

A patient with the Holmes-Adie syndrome had bilateral stellate ganglionectomy with benefit to visual accommodation and no ill effects. A later return of sympathetic innervation to the left eye was treated by superior cervical ganglionectomy, i.e., post-ganglionic sympathectomy.

Parotid pain associated with reflex salivation 
appeared in two weeks. Investigations are reported suggesting that pain was due to reflex vasodilatation. It could not be determined whether this was effected by vasodilator nerves or by release of bradykinin from gland cells rendered hypersensitive by sympathetic denervation but the evidence favoured the former theory.

The original pre-ganglionic sympathectomy was carried out while the patient was under the care of Dr. J. Marshall. Mr. I. C. S. Knight kindly cannulated the parotid ducts and the bradykinin estimation was by Dr. W. E. Brocklehurst. J. A. S. was in receipt of a grant from the Secretary of State for Scotland on the recommendation of the Advisory Committee for Medical Research.

\section{REFERENCES}

Barcroft, J. (1914). The Respiratory Function of the Blood. University Press, Cambridge.

Bernard, C. (1858). C. R. Acad. Sci. (Paris), 47, 245.

Burgen, A. S. V., and Emmelin, N. G. (1961). Physiology of the Salivary Glands. Arnold, London.
Carlson, A. J. (1907). Amer. J. Physiol., 19, 408.

Collier, H. O. J., and Shorley, P. G. (1960). Brit. J. Pharmacol., 15, 601.

Emmelin, N. (1955). Acta physiol. scand., 34, 29.

, and Engström, J. (1960). Lancet, 2, 263.

Folkow, B., and Laage-Hellman, J. E. (1960). Cited by Burgen and Emmelin (1961). Personal communication. P. 65.

Frey, L. (1923). Rev. neurol., 2, 97.

Gardner, W. J., and Abdullah, A. F. (1955). Amer. J. med. Sci., 230, 65.

Glaister, D. H., Hearnshaw, J. R., Heffron, P. F., Peck, A. W., and Patey, D. H. (1958). Brit. med. J., 2, 942.

Heidenhain, R. (1872). Pflügers Arch. ges. Physiol., 5, 309.

Henderson, V. E., and Loewi, O. (1905). Nauny Schmiedeberg's Arch. exp. Path. Pharmak., 53, 62.

Hilton, S. M., and Lewis, G. P. (1956). J. Physiol., 134, 471.

Kidd, H. A. (1955). Brit. med. J., 2, 1309.

Kimura, E. T., Young, P. R., and Richards, R. K. (1960). J. Allergy, 31, 237.

Lowther, C. P., and Turner, R. W. D. (1960) Brit med. J., 2, 1049. Reichert, F. L., and Poth, E. J. (1933). Proc. Soc. exp. Biol. (N.Y.), 30, 973.

Russell, G. F. M. (1956). J. Neurol. Neurosurg. Psychiat., 19, 289.

Terroux, K. G., Sekelj, P., and Burgen, A. S. V. (1959). Canad. J. ڤి Biochem., 37, 5.

Tracy, G. D., and Cockett, F. B. (1957). Lancet, 1, 12.

Ungar, G., and Parrot, J-L. (1936). C. R. Soc. Biol. (Paris), 122, 1052.

Uprus, V., Gaylor, J. B., and Carmichael, E. A. (1934). Brain, 57, 443. $\vec{\omega}$

Wilson, W. C. (1936). Clin. Sci., 2, 273. 